\title{
Massive Star Forming Regions in the Galactic Plane: A Comparative Study Using BGPS, Spitzer, \& Optical/Near-IR Surveys
}

\author{
Guy S. Stringfellow ${ }^{1} \&$ the BGPS team \\ ${ }^{1}$ CASA, University of Colorado, 389 UCB, Boulder, CO, USA, Guy.Stringfellow@colorado.edu
}

\begin{abstract}
The Bolocam Galactic Plane Survey (BGPS) is a $1.1 \mathrm{~mm}$ continuum survey that has detected more than 8300 clumps over a 170 square degree survey area in the Galactic plane. The full power of these data is realised only when considering the full complement of data spanning millimetre through x-ray wavelengths.
\end{abstract}

Keywords. Galaxy: structure, ISM: jets and outflows, ISM: structure, stars: formation, surveys

The search for highly obscured star forming regions has become possible through longwavelength, large area, Galactic plane surveys using Spitzer and ground-based submillimeter (ATLASGAL; Schuller et al. 2009) and millimetre (Bolocam Galactic Plane Survey (BGPS); Aguirre et al. 2009 in press) surveys. BGPS is a $1.1 \mathrm{~mm}$ continuum survey that is contiguous over the range $-10.5^{\circ} \leqslant l \leqslant 90.5^{\circ}$ with $|b| \leqslant 0.5^{\circ}$ and $75.5^{\circ} \leqslant l \leqslant 87.5^{\circ}$ with $|b| \leqslant 1.5^{\circ}$. The BGPS survey has detected more than 8300 clumps over the entire 170 square degree survey area to a limiting non-uniform $5 \sigma$ noise level in the range 30 to $60 \mathrm{mJy} /$ beam (Rosolowsky et al. 2009). These clumps are believed to represent the earliest stages in the formation of massive stars.

For comparative analysis, Figure 1a shows a $\sim 32^{\prime}$ field-of-view of the BGPS survey centred on the DR21 and W75 complexes. The $1.1 \mathrm{~mm}$ emission traces active regions of cold, dense gas being heated by embedded massive stars, and matches well with the IRS and ERO objects.

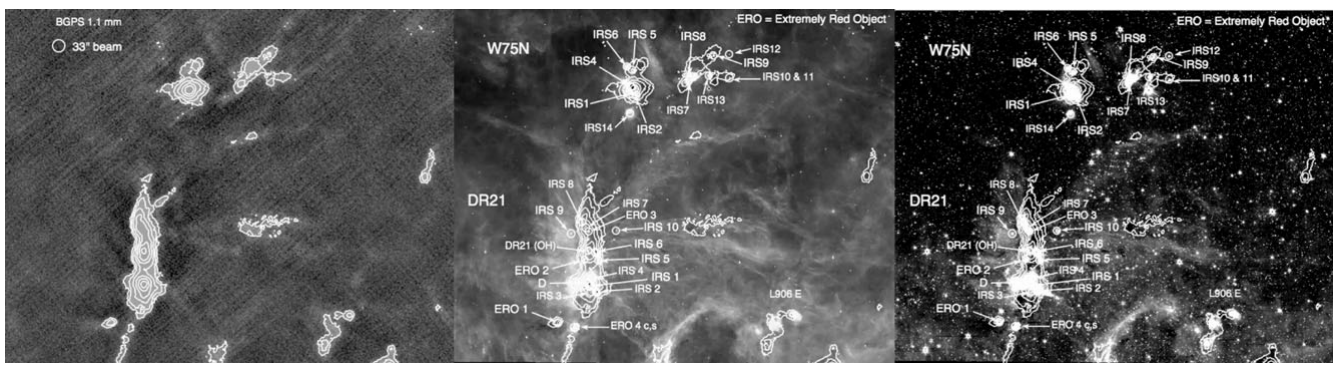

Figure 1. DR21/W75 massive star forming complexes: $1.1 \mathrm{~mm}$ dust continuum emission (left; contours: $0.2,0.44,0.96,2.1,4.6,10 \mathrm{Jy} /$ beam.), Spitzer $8 \mu \mathrm{m}$ (middle) and $4.5 \mu \mathrm{m}$ (right).

\section{References}

Aguirre, J. et al. 2009, ApJ Suppl in press

Rosolowsky, E. et al. 2009, ApJ Suppl in press

Schuller, F., Menten, K. M., Contreras, Y., et al. 2009, A\&GA in press 